\title{
Application of Wide Area Measurement Systems to Islanding Detection of Bulk Power Systems
}

\author{
Zhenzhi Lin, Tao Xia, Yanzhu Ye, Ye Zhang, Lang Chen, Yilu Liu, Fellow, IEEE, Kevin Tomsovic, Fellow, IEEE, \\ Terry Bilke, and Fushuan Wen
}

\begin{abstract}
A fast islanding detection tool can help power dispatchers monitor and control power system operations. Frequency monitoring network (FNET) is a low cost and quickly deployable wide-area phasor measurement system at the distribution system level. The frequency disturbance recorder (FDR) in FNET is actually a single-phase phasor measurement unit (PMU) installed at ordinary $120 \mathrm{~V}$ outlets in the sense that it measures the voltage phase angle, amplitude, and frequency from a single-phase voltage source. Based on the data collected by the FDRs deployed in the North American power grid, two islanding detection methods, the frequency difference method and the change of angle difference method, are proposed. The nine real cases recorded, including islanding cases, generation trip cases, load shedding cases and oscillation cases, are presented to verify the proposed methods of islanding detection. Sensitivity analysis on the thresholds of the frequency deviation and angle deviation is done based on the real measurement data for obtaining the insensitive interval of two thresholds. The results show that the proposed methods can correctly detect power system islanding, and will not be falsely triggered by generation trips, load shedding and system oscillations.
\end{abstract}

Index Terms-Event detection, frequency disturbance recorder, islanding detection, phasor measurement unit, wide area measurement system.

\section{INTRODUCTION}

$\mathbf{W}$ IDE area measurement systems (WAMS) have made possible the monitoring of entire bulk power systems as well as provided insights into system dynamics. Frequency monitoring network (FNET) is a low cost and quickly deployable wide-area frequency measurement system with high dynamic accuracy and minimal installation cost. A type of single-phase phasor measurement unit (PMU) known as a

Manuscript received May 01, 2012; revised November 23, 2012; accepted February 26, 2013. Date of publication March 20, 2013; date of current version April 18, 2013. This work was supported in part by the NSF, the Midwest ISO, and the National Natural Science Foundation of China (51007080). Paper no. TPWRS-00458-2012.

Z. Lin is with the School of Electrical Engineering, Zhejiang University, Hangzhou 310027, China, and also with the University of Tennessee, Knoxville, TN 37996 USA (e-mail: zhenzhi.lin@gmail.com).

T. Xia is with Dominion Virginia Power, Richmond, VA 23219 USA (e-mail: tao.xia@dom.com).

Y. Ye, Y. Zhang, L. Chen, Y. Liu, and K. Tomsovic are with the University of Tennessee, Knoxville, TN 37996 USA (e-mail: yye2@utk.edu; zoe.yezhang@gmail.com; lchen20@utk.edu; liu@utk.edu; tomsovic@eecs. utk.edu).

T. Bilke is with the Midwest ISO, Carmel, IN 46032 USA (e-mail: tbilke@midwestiso.org).

F. Wen is with the School of Electrical Engineering, Zhejiang University, Hangzhou 310027, China (e-mail: fushuan.wen@gmail.com).

Color versions of one or more of the figures in this paper are available online at http://ieeexplore.ieee.org.

Digital Object Identifier 10.1109/TPWRS.2013.2250531 frequency disturbance recorder (FDR) is installed at ordinary $120 \mathrm{~V}$ outlets and is used in FNET to collect voltage, angle, and frequency measurements at the $120 \mathrm{~V}$ distribution system level. These measurements are then transmitted across the Internet to a central location, where they are synchronized, analyzed, and archived. More information about FNET can be found in [1]-[4].

Event detection is a fundamental application of "smart grid" technologies for the transmission system. Power system events such as generator trips, line trips, load shedding, islanding, and oscillations create perturbations in the voltage, frequency, and angles. These disturbances propagate throughout the electrical network in time and space. Using time-synchronized phasor and frequency measurements, it is possible to detect and locate these events in near real-time. Among these power system disturbances, islanding is the situation in which a part of the grid becomes electrically isolated from the remainder of the power system.

There are various issues associated with unintentional islanding. The power system will undergo severe frequency and power angle swings in the islanded region. The severity of the swings is proportional to the generation and load imbalance in the island. Failure to stabilize the frequency can lead to collapse of the island and delay restoration. It is not unusual for a distributed generation (DG) system to be separated from an interconnection and become an island. This makes it imperative for a DG system to have islanding detection and protection mechanisms. Methods commonly utilized to date for a distribution system with DG can be classified in three main categories [5]. They are passive methods (such as over/under voltage method, over/under frequency method), active methods (such as active frequency drifting method and phase-shift method [6]-[8]), and communication based methods (such as power-line-signaling-based scheme [9], [10]). Large scale power system islanding is rare. Even so, since large systems are generally controlled by multiple operators, it is important to give all dispatchers a common wide-area view of the network. In [11]-[16], a controlled islanding algorithm based slow coherency theory is proposed. In [17]-[20], a splitting strategy for power systems is presented based on ordered binary decision diagrams (OBDD). In [21], a splitting strategy of large scale power system islanding based on slow coherency and angle modulated particle swarm optimization is given.

Islanding visualization techniques are very straightforward. A quick view of two sets of frequency traces will show system separation. The frequency can be graphed, or the phase angle plotted, to show that the systems have become islanded. However, methods commonly utilized to date focus on the DG islanding detection in the distribution systems, and how to 


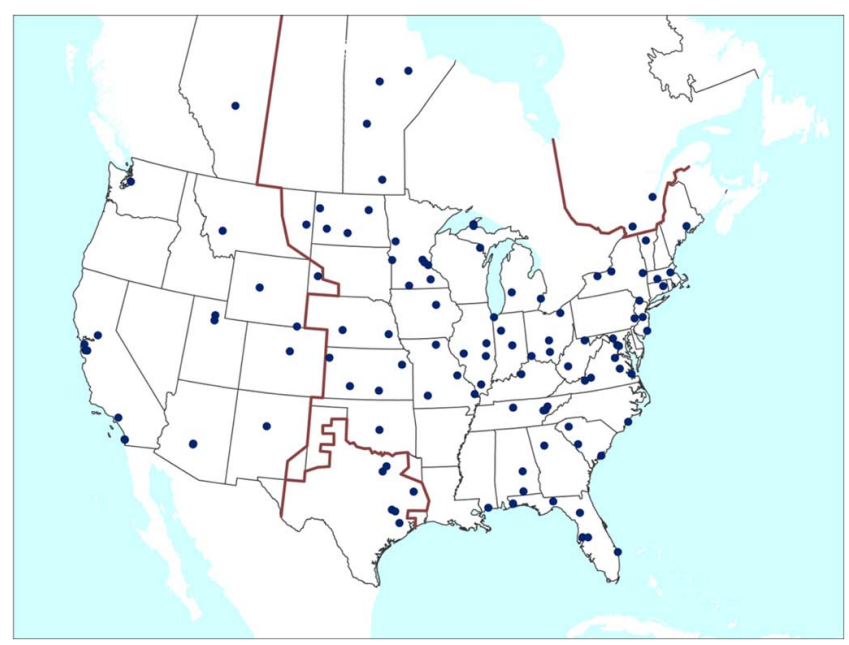

Fig. 1. Map of FDR locations in North America.

divide or split the islanding of large scale power system for preventing a big blackout before the disturbances occur. They cannot present a real-time wide-area view of the islanding. Computer methods for detecting islanding are not as simple as viewing a plot, but they can be performed quickly and with high confidence. Computer detection also enables alarming which can be used to alert operators and engineers.

The FNET system was initially deployed in 2004. Since then, more than 80 FDRs units have been deployed in the three North American interconnections: Eastern Interconnection (EI), Western Electricity Coordinating Council system (WECC), and Electric Reliability Council of Texas system (ERCOT). Fig. 1 shows the FDR locations in North America. With these FDRs, islanding can be detected in near real time. The current approaches used in FNET are the frequency difference method and the change of angular difference method. Although FNET uses FDRs to collect frequency and angle data, the techniques presented here are equally applicable to PMU data as well.

This paper describes the frequency difference method and the change of angle difference method for detecting power system islanding in FNET and the results of nine real recorded case studies on the North American interconnections. Based on the real measurement data, the sensitivity analysis on the thresholds of the frequency deviation and angle deviation is performed, and then the insensitive interval of two thresholds is obtained. This helps to set reasonable thresholds of detecting islanding. These results provide a detailed view of one component of an advanced intelligent event-detection system for the future power grid.

\section{ISLANDING Detection Methods}

As noted earlier, FNET uses both the frequency difference method and the change of angle difference method to detect islanding. The following outlines the methods.

\section{A. Frequency Difference Method}

To demonstrate the utility of distribution level wide-area measurements, system frequency is of primary importance at all voltage levels in the interconnection. The frequency variation method FNET uses to detect islanding is to locate the FDRs which the frequency difference between FDRs and the

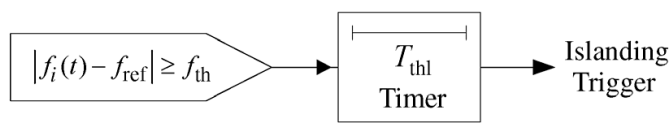

Fig. 2. Process of the frequency difference method.

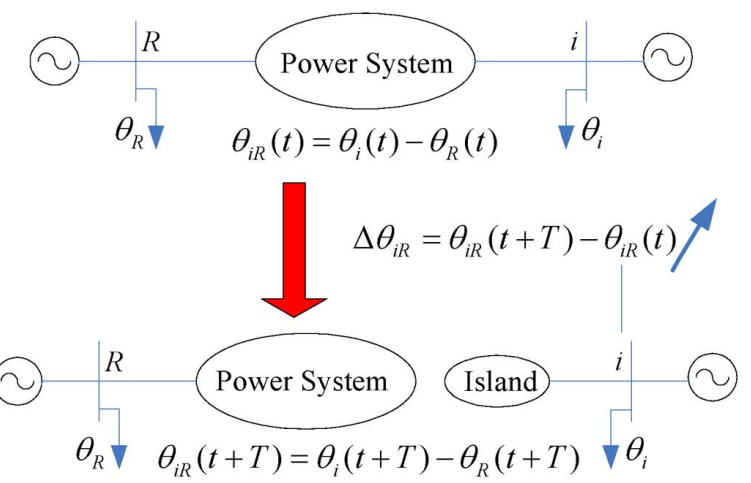

Fig. 3. Simple islanding case of power system.

reference FDR exceeds the threshold for a period of time. This can be represented as

$$
\left\{\begin{array}{l}
\left|f_{i}(t)-f_{\mathrm{ref}}\right| \geq f_{\mathrm{th}}, \forall t \in\left[T_{m}, T_{n}\right] \\
T_{n}-T_{m}>T_{\mathrm{th} 1}
\end{array}\right.
$$

where $f_{i}(t)$ is the frequency value measured in the time $t$ by the $i$ th FDR of FNET, $f_{\text {ref }}$ the reference frequency value, $f_{\text {th }}$ the threshold value for frequency difference, and $T_{\text {th1 }}$ the threshold value for the duration of time $\left[T_{m}, T_{n}\right]$. Fig. 2 shows the process of the frequency difference method.

In this paper, the median frequency of all the FDRs is taken as the reference $f_{\text {ref }}$; and according to experience, $f_{\text {th }}$ and $T_{\text {th1 }}$ are set to 20 milli- $\mathrm{Hz}(\mathrm{mHz})$ and 3 seconds, respectively.

\section{B. Change of Angle Difference Method}

The FNET system also uses the change in the angle for detecting islanding. The initial phase angle difference is periodically updated during steady state conditions. A time interval for phase angle comparison is needed to allow the separated system to accumulate an angle difference. The FNET angle variation method can be represented as

$$
\left\{\begin{array}{l}
\left|\theta_{i R}(t+T)-\theta_{i R}(t)\right| \geq \theta_{\mathrm{th}}, \forall t \in\left[T_{m}, T_{n}\right] \\
T_{n}-T_{m}>T_{\mathrm{th} 2}
\end{array}\right.
$$

where $\theta_{i R}(t)$ is the angle value between bus $i$ and $R$ measured in the time $t, T$ the time interval for the phase angle comparison, $\theta_{\mathrm{th}}$ the threshold value for angle difference, and $T_{\mathrm{th} 2}$ the threshold value for the duration of time $\left[T_{m}, T_{n}\right]$.

A simple case of power system is shown in Fig. 3 to illustrate the change in angle difference method.

It can be seen from Fig. 3 that the change of angle difference between FDRs and the reference will increase when islanding occurs. Fig. 4 shows the process of the change of angle difference method. In this paper, $\theta_{\mathrm{th}}$ is set to 30 degrees $(0.5236 \mathrm{rad})$; and according to experience, both $T$ and $T_{\mathrm{th} 2}$ are set to 3 seconds.

The FNET islanding detection program uses both of the proposed methods to detect islanding events. An islanding detection algorithm should discriminate islanding from other grid disturbances. The following real case studies were used to verify 


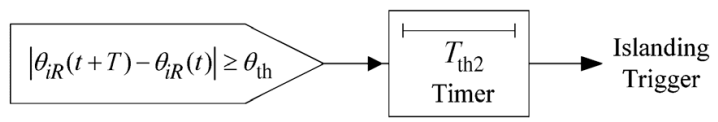

Fig. 4. Process of the change of angle difference method.

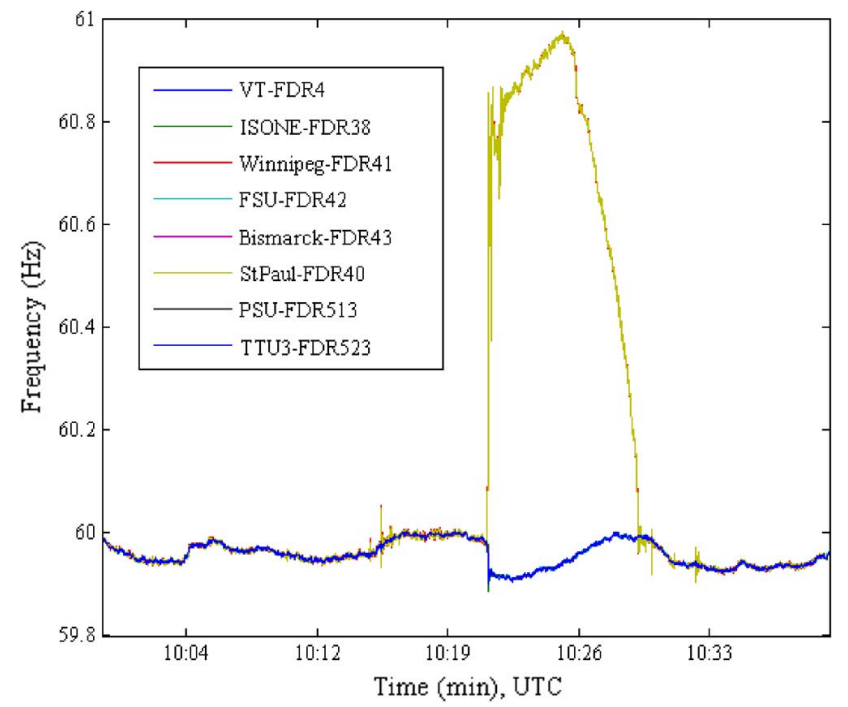

Fig. 5. Frequency plot of case 1.

the FNET islanding detection does not produce false triggers. The sensitivity of the threshold values is discussed based on the measurement data.

\section{CASE StUdies}

Since the FNET system was initially deployed in 2004, it has detected three actual islanding cases in North America power grid. In the following, three islanding cases and another six cases are presented to verify the proposed methods and to direct the selection of the threshold values:

Case 1: EI Islanding at 09/18/2007 10:21:23 UTC

Case 2: WECC islanding at 06/01/2010 23:37:32 UTC

Case 3: WECC islanding at 07/22/2010 21:38:09 UTC

Case 4: Generation trip at 08/12/2010 20:44:03 UTC

Case 5: Load shedding at 08/12/2010 12:15:31 UTC

Case 6: A similar EI islanding at 11/09/2010 18:38:16 UTC

Case 7: System oscillation at 04/22/2011 15:53:15 UTC

Case 8: System oscillation at 04/24/2011 05:19:22 UTC

Case 9: Nuclear unit trip for Tornado at 04/27/2011 21:36:28 UTC

\section{A. Case 1: EI Islanding}

The islanding in case 1 occurred in the EI system of North America at 10:21:23 UTC (Coordinated Universal Time) on September 18, 2007. The islanding lasted about $9 \mathrm{~min}$. A 40-min plot of frequency and angle plot are shown in Figs. 5 and 6. It can be seen that the islanding occurred during 10:21 to 10:30, and the frequency and angle of three FDRs deviated from the other five FDRs in this interval. After 10:30, the frequency of three FDRs returned back to the values recorded by the other five FDRs, but the angles of three FDRs maintained a fixed difference to the other five FDRs.

The FNET program detected the islanded FDRs at Winnipeg, $\mathrm{MB}$, Bismarck, ND and St. Paul, MN in $3.8 \mathrm{~s}, 3.7 \mathrm{~s}$ and $3.7 \mathrm{~s}$

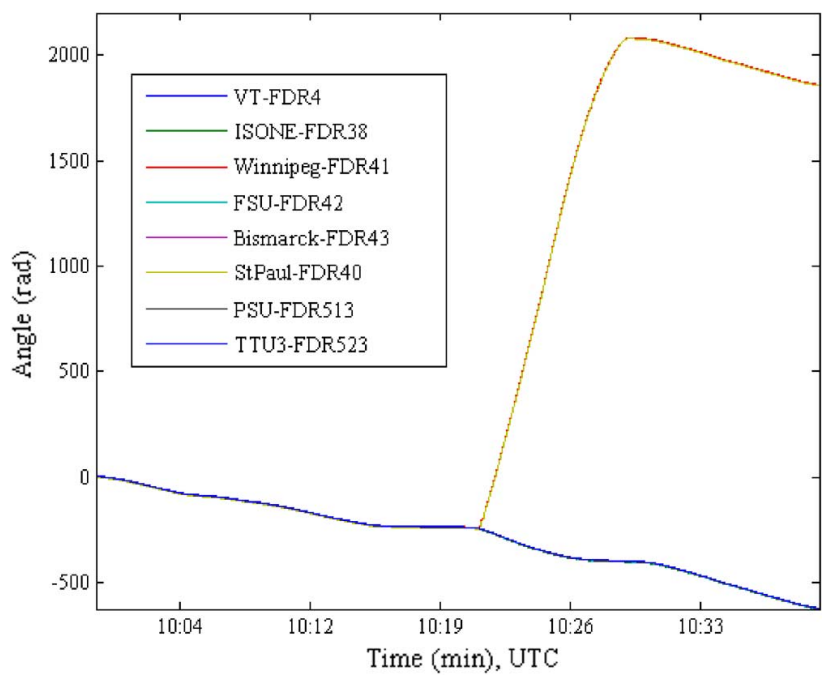

Fig. 6. Angle plot of case 1 .

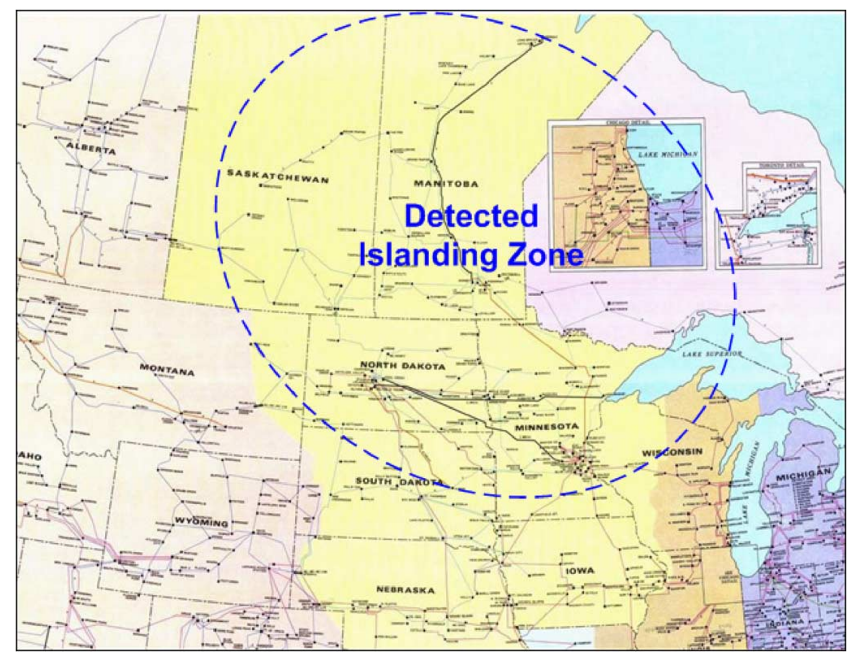

Fig. 7. Detected islanding zone of case 1 in North America power grid.

respectively by using the frequency difference method. The maximum frequency differences measured in Winnipeg, MB, Bismarck, ND and St. Paul, MN were 1.0431 Hz, $1.0577 \mathrm{~Hz}$ and $1.0577 \mathrm{~Hz}$, respectively. The time durations for which frequency differences were above the threshold were $498.2 \mathrm{~s}$, $498.3 \mathrm{~s}$, and $498.2 \mathrm{~s}$, respectively. The change of angle difference method also successfully detected the islanded FDRs at Winnipeg, MB, Bismarck, ND and St. Paul, MN in $3.6 \mathrm{~s}$, $3.2 \mathrm{~s}$, and $3.2 \mathrm{~s}$, respectively. The maximum changes of angle difference measured in Winnipeg, MB, Bismarck, ND and St. Paul, MN were $19.4321 \mathrm{rad}, 19.4299 \mathrm{rad}$ and $19.4299 \mathrm{rad}$, respectively. The time durations for which the changes of angle difference were above the threshold were $495.8 \mathrm{~s}, 498.1 \mathrm{~s}$ and $498.1 \mathrm{~s}$, respectively. Fig. 7 shows the detected islanding zone in North America power grid.

\section{B. Case 2: WECC Islanding One}

The islanding in case 2 occurred in the WECC system of North America at 23:37:32 UTC on June 1, 2010. The islanding lasted about $5 \mathrm{~min}$ and its 30-min frequency plot and angle 


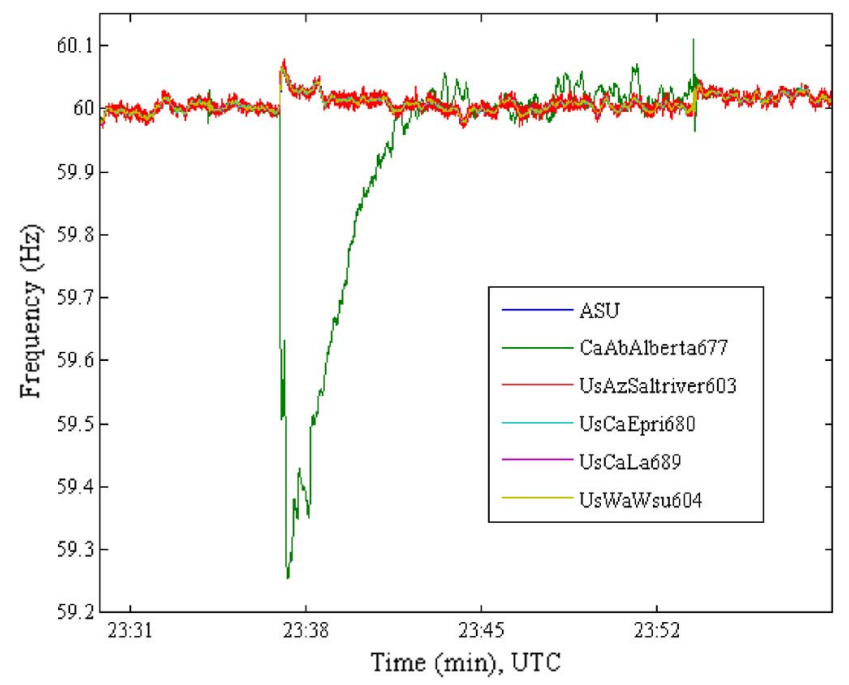

Fig. 8. Frequency plot of case 2.

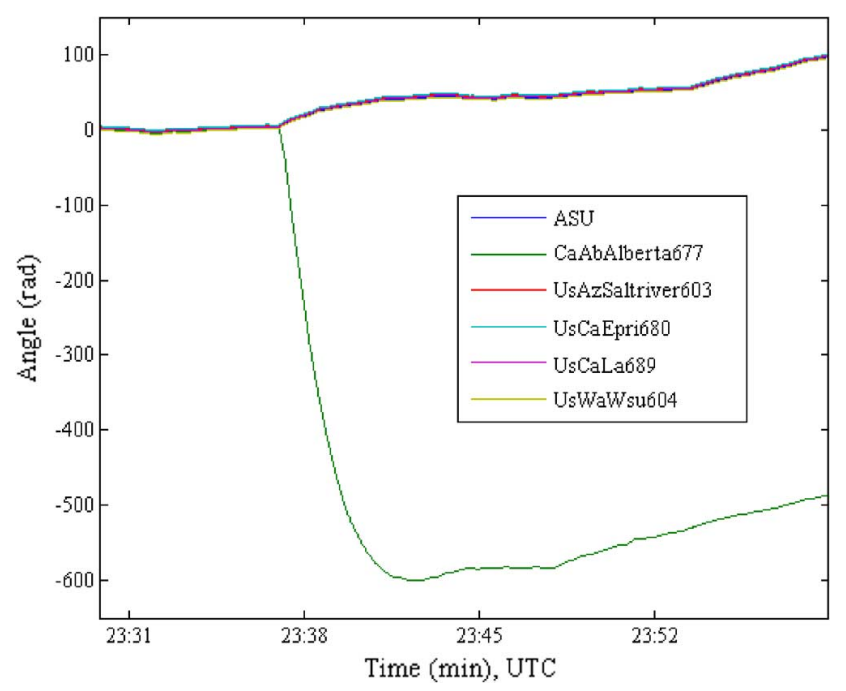

Fig. 9. Angle plot of case 2 .

plot are shown in Fig. 8 and Fig. 9. It can be seen that the islanding occurred during 23:37 to 23:42, and the frequency and angle of one FDR deviated from the other five FDRs in this interval. After 23:42, the frequency of the FDR returned back to the values recorded by the other five FDRs, but the angle of the FDR maintained a fixed difference to the other five FDRs.

The FNET program detected the islanded FDR at Alberta, $\mathrm{AB}$ in $3.5 \mathrm{~s}$, by using the frequency difference method. The maximum frequency difference measured in Alberta, AB was $0.7925 \mathrm{~Hz}$. The time duration for which the frequency difference was above the threshold was $281.1 \mathrm{~s}$, and was far greater than the time threshold. The change of angle difference method also successfully detected the islanded FDR at Alberta, AB in $3.3 \mathrm{~s}$. The maximum change of angle difference measured in Alberta, $\mathrm{AB}$ was $14.8595 \mathrm{rad}$. The time duration for which the change of angle difference was above the threshold was 280.9 s, and was also far greater than the time threshold. Fig. 10 shows the detected islanding zone in North America power grid.

\section{Case 3: WECC Islanding Two}

The islanding in case 3 occurred in the WECC at 21:38:09 UTC on July 22, 2010. The islanding lasted about 7 minutes and

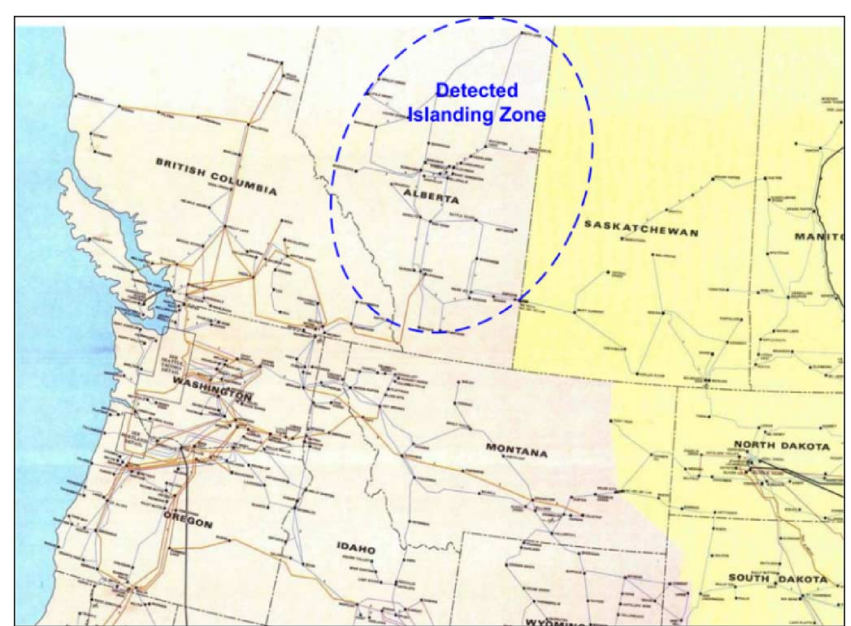

Fig. 10. Detected islanding zone of case 2 and 3 in North America power grid

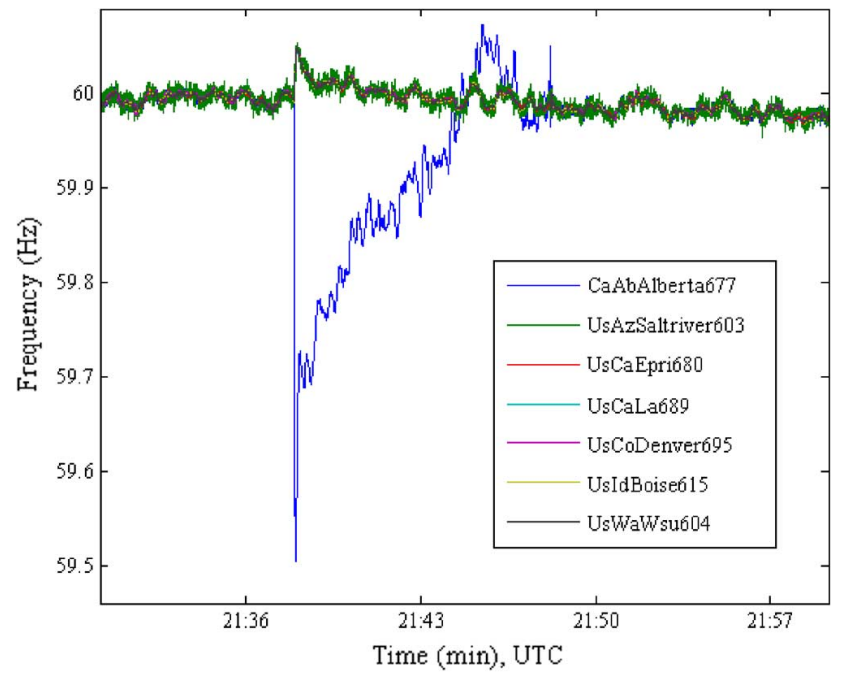

Fig. 11. Frequency plot of case 3 .

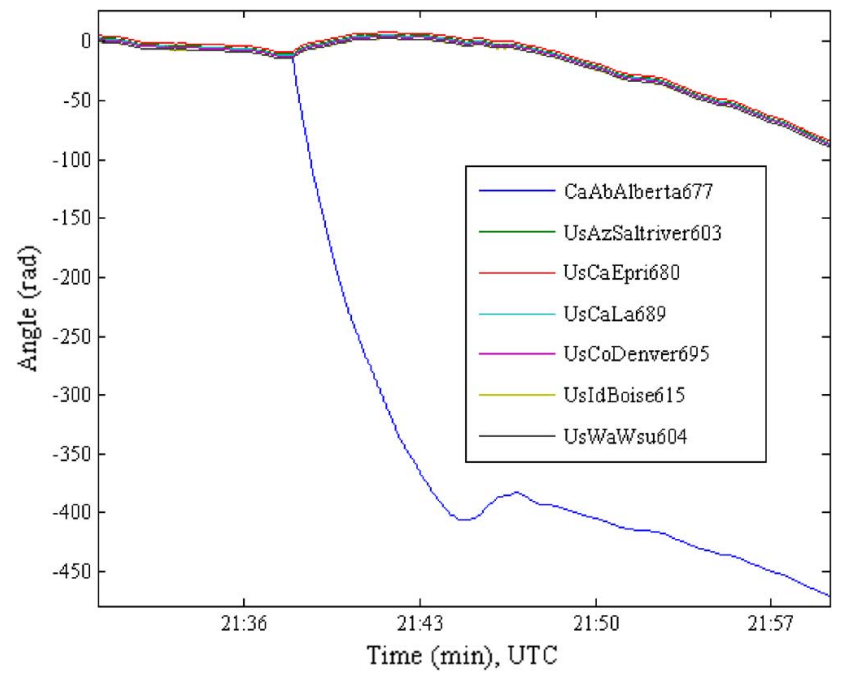

Fig. 12. Angle plot of case 3 .

its 30-min frequency plot and angle plot are shown in Figs. 11 and 12 . It can be seen that the islanding occurred during 21:38 to $21: 45$, and the frequency and angle of one FDR deviated from the other six FDRs in this interval. After 21:45, the frequency 


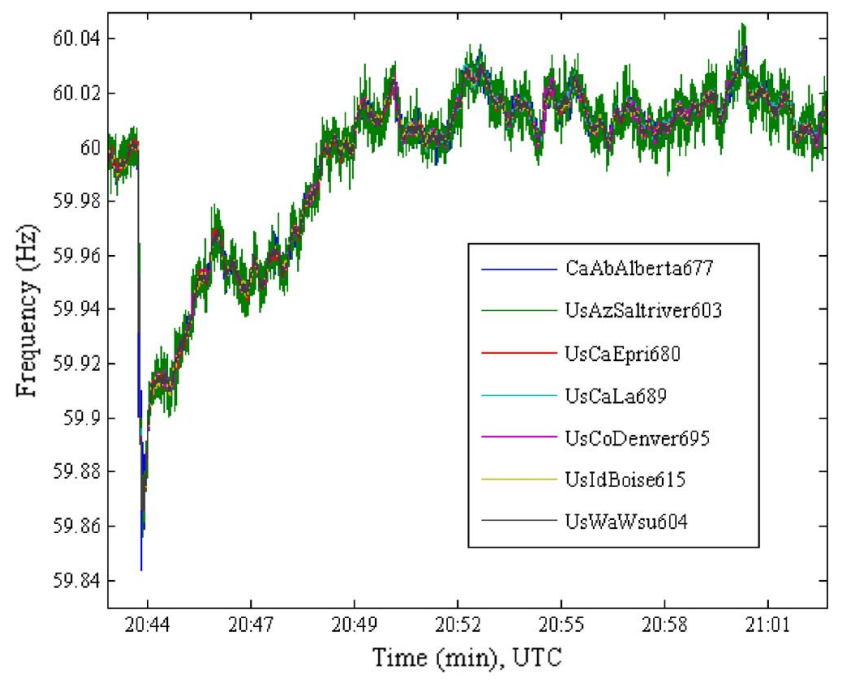

Fig. 13. Frequency plot in the generation trip case.

of the FDR returned back to the values recorded by the other six FDRs, but the angle of the FDR maintained a fixed distance to the other six FDRs.

The FNET program detected the islanded FDR at Alberta, $\mathrm{AB}$ in $3.7 \mathrm{~s}$, by using the frequency difference method. The maximum frequency difference measured in Alberta, $\mathrm{AB}$ was $0.5291 \mathrm{~Hz}$. The time duration for which frequency difference was above the threshold was $390.0 \mathrm{~s}$, and was far greater than the time threshold. The change of angle difference method also successfully detected the islanded FDR at Alberta, $\mathrm{AB}$ in $3.7 \mathrm{~s}$. The maximum change of angle difference measured in Alberta, $\mathrm{AB}$ was $9.6043 \mathrm{rad}$. The time duration for which the change of angle difference was above the threshold was $389.1 \mathrm{~s}$, and was far greater than the time threshold. The detected islanding zone in North America power grid can also be seen in Fig. 10.

\section{Case 4: Generation Trip}

Generation trips observed by FNET were used to check whether or not the program would falsely trigger by testing the frequency difference method and the change of angle difference method. A large generation trip occurred in the WECC at 20:44:03 UTC on August 12, 2010. The 30-min frequency plot and angle plot are shown in Figs. 13 and 14. It can be seen that the frequency and angle of all FDRs encountered a sudden drop at 20:44 and then the frequency returned back slowly, and the FDRs do not deviate from each other.

The offline simulation based on the real data recorded did not falsely trigger the islanding detection module. Though the maximum frequency difference was $56.4 \mathrm{mHz}$, which is above the threshold, the time duration of $1.6 \mathrm{~s}$ was below the time threshold. The maximum angle change was $0.5169 \mathrm{rad}$, which is below the threshold.

\section{E. Case 5: Load Shedding}

A load shedding event observed by FNET was also used to check whether or not the program will falsely trigger. The load shedding occurred in WECC at 12:15:31 UTC on August 12, 2010. The 30-min frequency plot and angle plot are shown in Figs. 15 and 16. It can be seen that the frequency and angle of

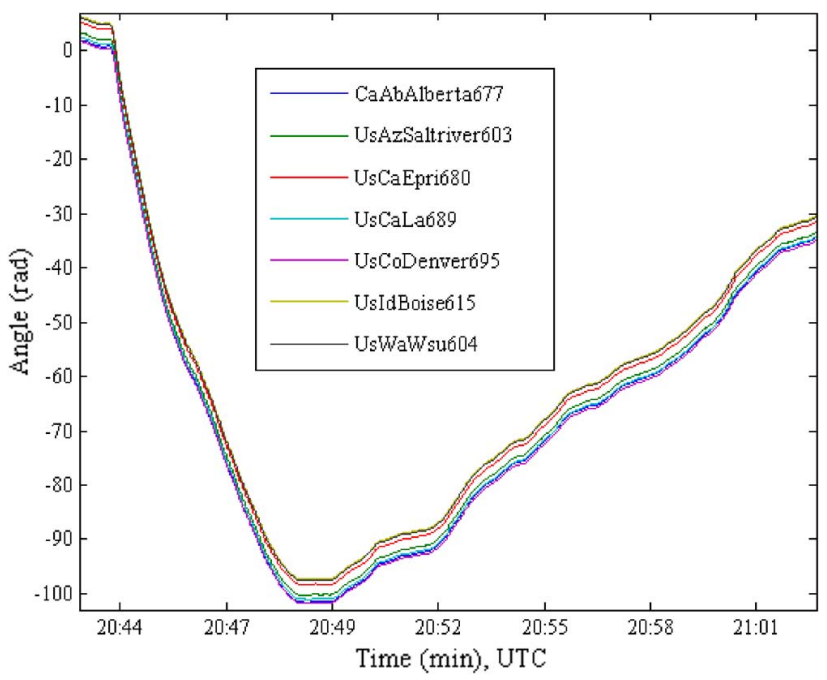

Fig. 14. Angle plot in the generation trip case.

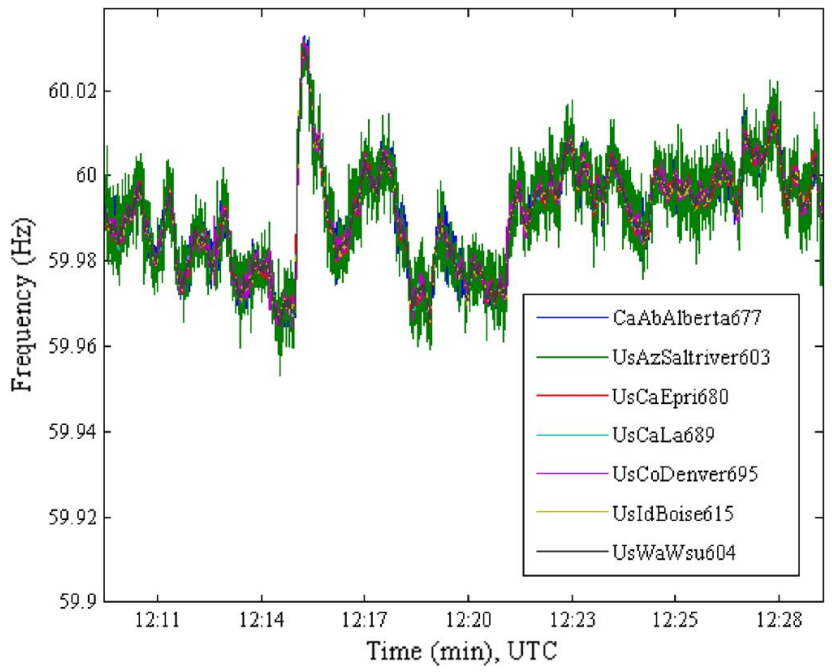

Fig. 15. Frequency plot in the load shedding case.

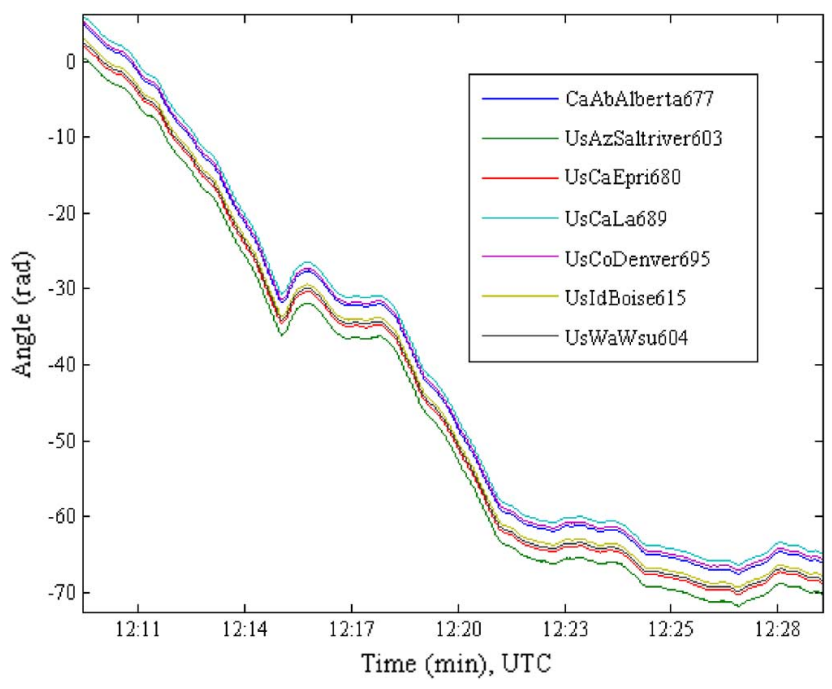

Fig. 16. Angle plot in the load shedding case.

all FDRs encountered a sudden rise at 12:15, and the FDRs do not deviate against each other. 


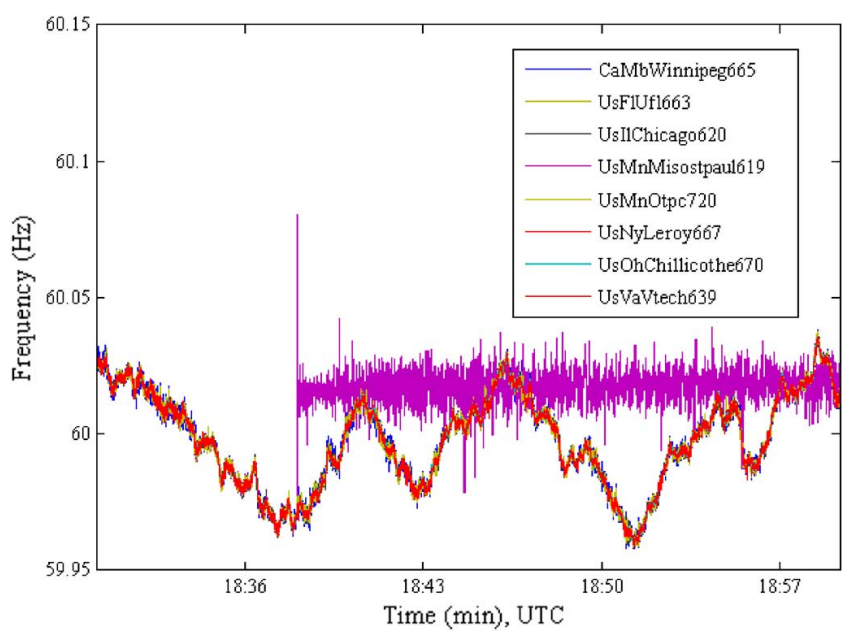

Fig. 17. Frequency plot of case 6 .

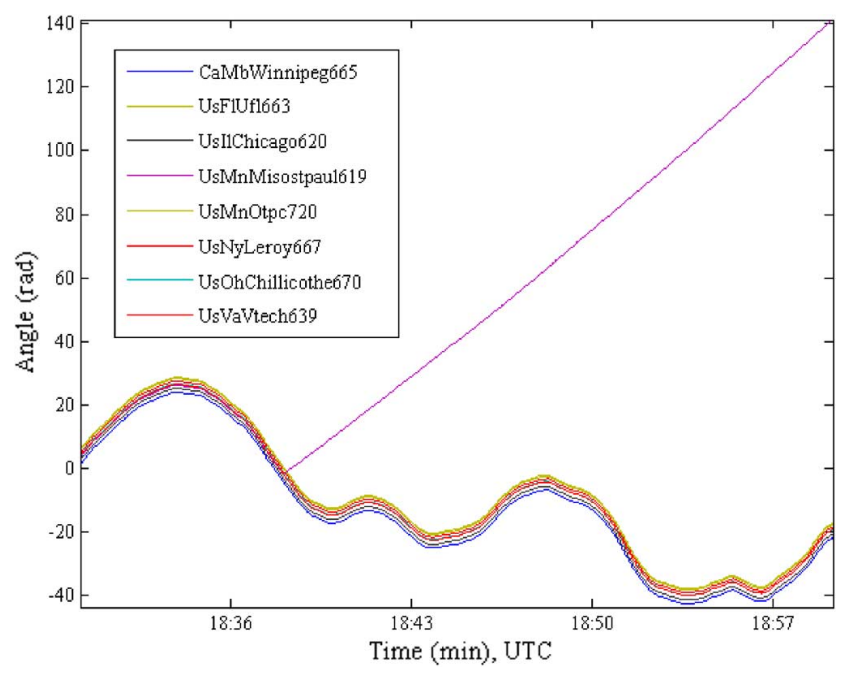

Fig. 18. Angle plot of case 6 .

The offline simulations based on the real data recorded did not falsely trigger the islanding detection module. Though the maximum frequency difference was $34.8 \mathrm{mHz}$, which is above the threshold, the time duration of the frequency difference was only $0.2 \mathrm{~s}$ which is below the time threshold. The maximum angle change of $0.0744 \mathrm{rad}$ was far less than the threshold.

\section{F. Case 6: A Similar Islanding in EI}

A similar islanding in case 6 occurred in EI at 18:38:16 UTC on November 9, 2010. In this case, the FDR at St. Paul, MN was separated from the other FDRs because it was supplied by a local diesel generator with more noise. As a result, this is a special case. To make the figure more readable, the 30-min frequency plot and angle plot of only 8 typical FDRs are shown in Figs. 17 and 18. It can be seen that the islanding occurred at 18:38, and the frequency and angle of seven FDRs deviated from the other FDR.

The FNET detection program detected the islanded FDR at St. Paul, $\mathrm{MN}$ in $3.3 \mathrm{~s}$, by using the frequency difference method and based on the real recorded data of 25 FDRs. The maximum frequency difference measured in St Paul, MN

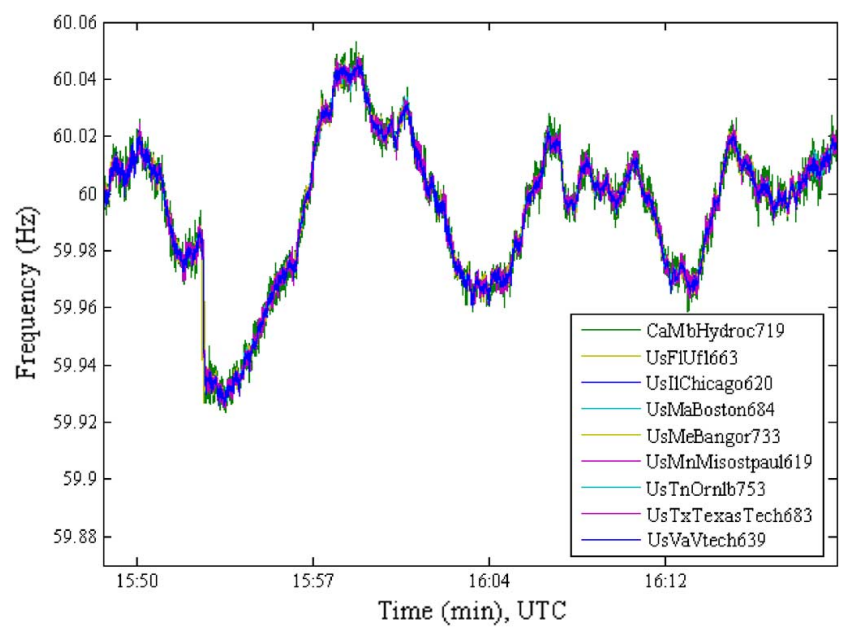

Fig. 19. Frequency plot of case 7.

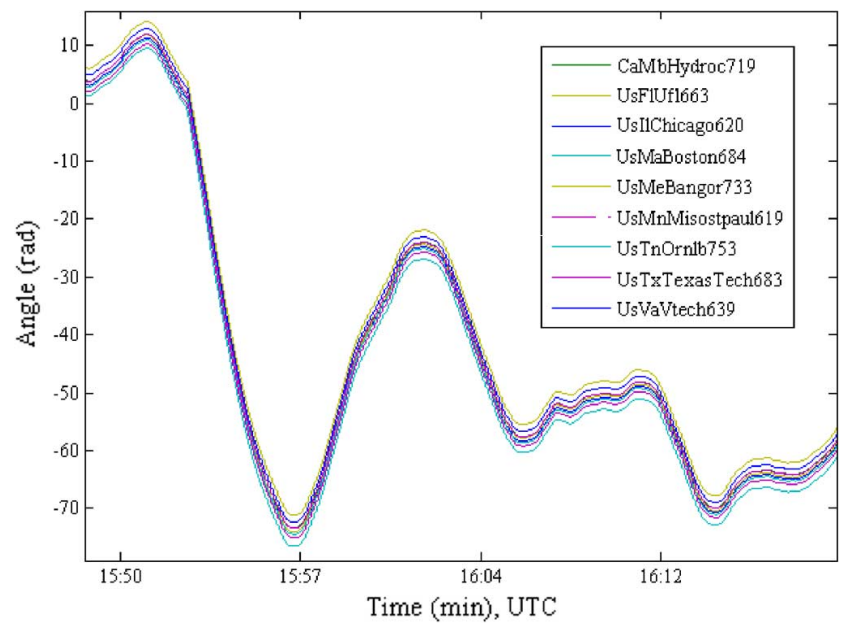

Fig. 20. Angle plot of case 7 .

was $0.1098 \mathrm{~Hz}$. The time duration for which the frequency difference was above the threshold was $138.6 \mathrm{~s}$. The change of angle difference method also successfully detected the islanded FDR at St. Paul, MN in $3.3 \mathrm{~s}$. The maximum change of angle difference measured in St. Paul, MN was 1.2045 rad. The time duration for which the change of angle difference was above the threshold was $158.3 \mathrm{~s}$.

\section{G. Case 7: System Oscillation One}

The system oscillations observed by FNET were used to check whether or not the program would falsely trigger by testing the frequency difference method and the change of angle difference method. The system oscillation in case 7 occurred in EI at 15:53:15 UTC on April 22, 2011. To make the figure more readable, the 30-min frequency plot and angle plot of only 9 typical FDRs are shown in Figs. 19 and 20. It can be seen that the frequency and angle of all FDRs oscillated together and do not deviate from each other.

In the offline simulation of this case, the FNET program detected the islanded FDRs at Boston, MA and Bangor, ME by using the frequency difference method based on the real recorded data of 37 FDRs. The maximum frequency differences measured in Boston, MA and Bangor, ME were $0.0413 \mathrm{~Hz}$ 


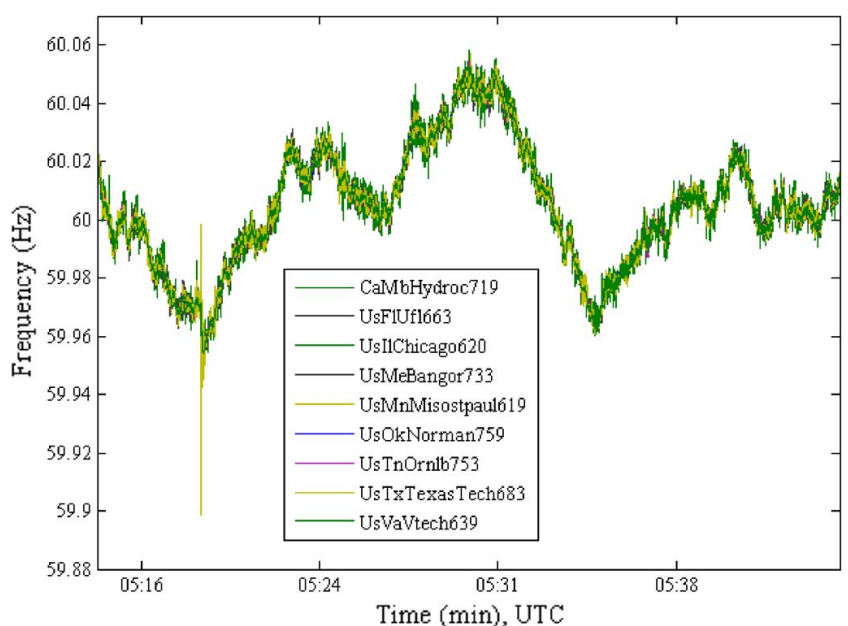

Fig. 21. Frequency plot of case 8 .

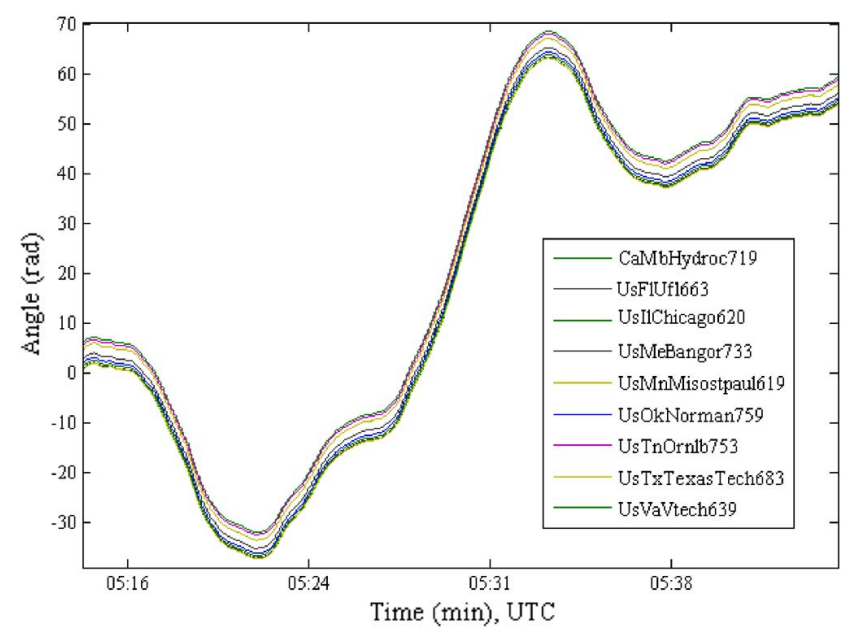

Fig. 22. Angle plot of case 8 .

and $0.0396 \mathrm{~Hz}$, respectively. The time durations for which frequency differences were above the threshold were both $3 \mathrm{~s}$. However, the islanding detection module was not falsely triggered by using the change of angle difference method. The maximum angle changes measured in Boston, MA and Bangor, ME were $0.0413 \mathrm{rad}$ and $0.0396 \mathrm{rad}$, which are below the threshold. As a result, the system oscillation in case 7 does not trigger the FNET islanding detection module because a combination of the proposed two methods is used in FNET.

\section{H. Case 8: System Oscillation Two}

The system oscillation in case 8 occurred in EI at 05:19:22 UTC on April 24, 2011. To make the figure more readable, the 30 -min frequency plot and angle plot of only 9 typical FDRs are shown in Figs. 21 and 22. It can be seen that the frequency and angle of all FDRs oscillated together and do not deviate from each other.

The offline simulations based on the real recorded data of 39 FDRs did not falsely trigger the islanding detection module. Though the maximum frequency difference was $72.5 \mathrm{mHz}$,

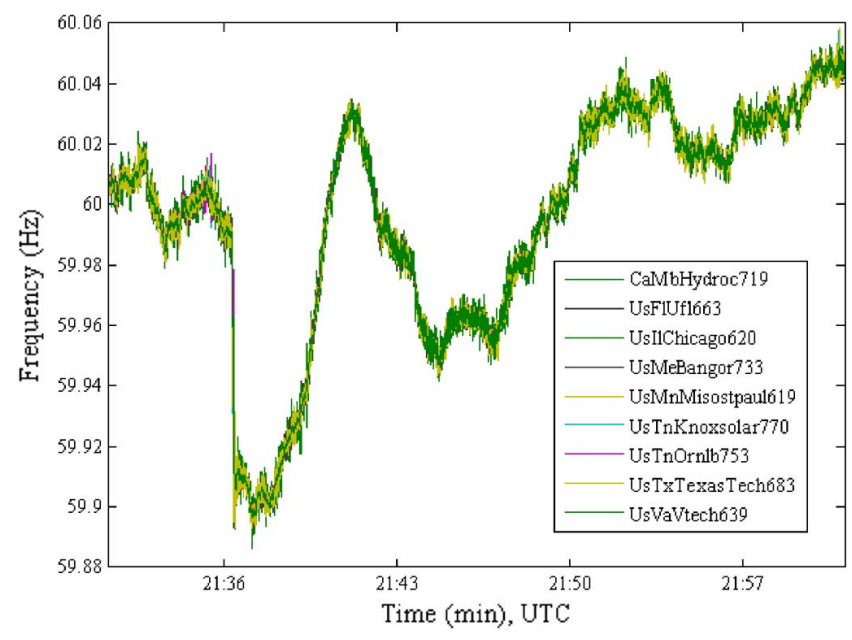

Fig. 23. Frequency plot of case 9 .

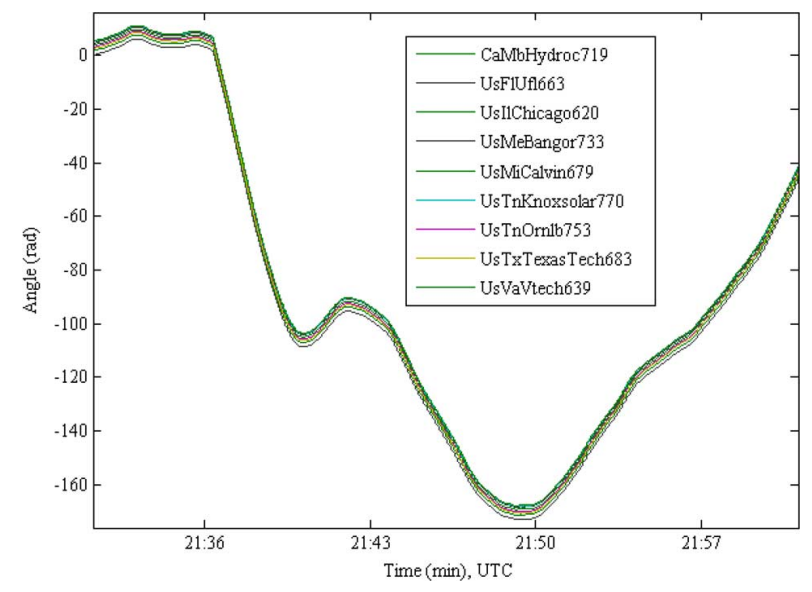

Fig. 24. Angle plot of case 9.

which is above the threshold, the time duration of $1.1 \mathrm{~s}$ was below the time threshold. The maximum angle change was $0.4011 \mathrm{rad}$, which is below the threshold.

\section{Case 9: Nuclear Unit Trip for Tornado}

The Tornado disturbance in case 9 occurred in EI at 21:36:28 UTC on April 27, 2011. In this case, a mega storm hit the South and Midwest United States with heavy flooding, lightning, rainfall, hailstorms and number of tornadoes. TVA lost all 3 Browns Ferry Nuclear units totaling approximately $3500 \mathrm{MW}$ that caused a frequency excursion to $59.90 \mathrm{~Hz}$. The system frequency recovered after $10 \mathrm{~min}$. To make the figure more readable, the 30-min frequency plot and angle plot of only 9 typical FDRs are shown in Figs. 23 and 24. It can be seen that the frequency and angle of all FDRs oscillated together and do not deviate from each other.

The offline simulations based on the real recorded data of 38 FDRs did not falsely trigger the islanding detection module. Though the maximum frequency difference was $59.4 \mathrm{mHz}$, which is above the threshold, the time duration of $0.7 \mathrm{~s}$ was below the time threshold. The maximum angle change was $0.2432 \mathrm{rad}$, which is below the threshold. 
TABLE I

Results of IsLANDing Detection in Nine ReAL CASES

\begin{tabular}{|c|c|c|c|c|c|}
\hline Case & $\begin{array}{c}\text { FDR } \\
\text { Location }\end{array}$ & $\begin{array}{c}\Delta f_{\max } \\
(\mathrm{Hz})\end{array}$ & $\begin{array}{c}\left(t_{\Delta f \geq f_{\text {th }}}\right)_{\max } \\
(\mathrm{s})\end{array}$ & $\begin{array}{c}\left|\theta_{t+T}-\theta_{t}\right|_{\max } \\
(\mathrm{rad})\end{array}$ & $\begin{array}{c}\left(t_{\Delta \theta \geq \theta_{\text {th }}}\right)_{\max } \\
(\mathrm{s})\end{array}$ \\
\hline \multirow{2}{*}{1} & Winnipeg,MB & 1.0431 & 498.2 & 19.4321 & 495.8 \\
& Bismarck,ND & 1.0577 & 498.3 & 19.4299 & 498.1 \\
& St. Paul,MN & 1.0577 & 498.2 & 19.4299 & 498.1 \\
\hline 2 & Alberta,AB & 0.7925 & 281.1 & 14.8595 & 280.9 \\
\hline 3 & Alberta,AB & 0.5291 & 390.0 & 9.6043 & 389.1 \\
\hline 4 & Alberta,AB & 0.0564 & 1.6 & 0.5169 & 0 \\
\hline 5 & SaltRiver,AZ & 0.0348 & 0.2 & 0.0744 & 0 \\
\hline 6 & St. Paul,MN & 0.1098 & 138.6 & 1.2045 & 158.3 \\
\hline 7 & Boston,MA & 0.0413 & 3.0 & 0.4826 & 0 \\
\hline \multirow{2}{*}{8} & Bangor,ME & 0.0396 & 3.0 & 0.4504 & 0 \\
\hline 9 & Norman,OK & 0.0224 & 0.5 & 0.1523 & 0 \\
\hline & Knoxas,TX & 0.0725 & 1.1 & 0.4011 & 0 \\
\hline
\end{tabular}

\section{Discussion}

The islanding detection results are summarized in Table I. The results show that both the frequency difference and the change of angle difference in the islanding events (case 1-3 and 6) exceed the threshold, and their duration of time is also above the time threshold. Thus, it can be concluded that the islanding can be correctly detected by the proposed methods. The results also show that the time durations in the generation trip (case 4 and 9), load shedding (case 5), and oscillation (case 8) events are below the time threshold, and the maximum change of angle difference is also below the threshold. It can be seen that though the system oscillation in case 7 is detected as islanding by using the frequency difference method, it is not detected by using the change of angle difference method, and then not triggered by the islanding detection program of FNET because both methods are jointly used in FNET to detect islanding. As a result, it can also be concluded that the generation trip, load shedding and oscillation events do not falsely trigger the islanding detection program.

It can be seen from Table I that if the threshold of the frequency deviation is set to more than $0.0725 \mathrm{~Hz}$, the cases 4, 5, 7 and 8 will not trigger the frequency difference method and will not be detected as islanding events; if the threshold of the angle deviation is set to more than $0.5169 \mathrm{rad}$, the cases $4,5,7$ and 8 will not trigger by the change of the angle difference method and will not be detected as islanding events. Conversely, if the threshold of the frequency deviation is set to less than 0.1098 $\mathrm{Hz}$, cases $1-3$ and 6 might be triggered by the frequency difference method and might be detected as the islanding events; if the threshold of angle deviation is set to less than $1.2045 \mathrm{rad}$, the cases 1-3 and 6 might be triggered by the change of the angle difference method and might be detected as the islanding events. Based the above analysis on the results of nine real cases, it is difficult to determine a precise sensitivity of the thresholds and timers on the results of the islanding detection. As a result, it is required that the sensitivity analysis on these threshold values is done by assuming a reasonable selection of the thresholds and timers based on experience.

Figs. 25-28 show the results of the sensitivity analysis on the thresholds of the frequency and angle deviations for these nine real cases. Figs. 25 and 26 illustrate the thresholds of the frequency deviation and angle deviation against the time of the de-

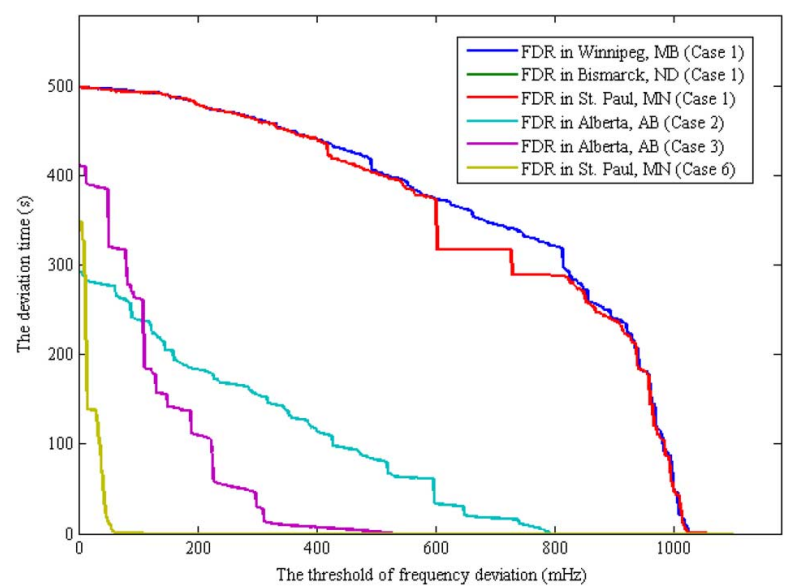

Fig. 25. Sensitivity analysis on the upper threshold of the frequency deviation.

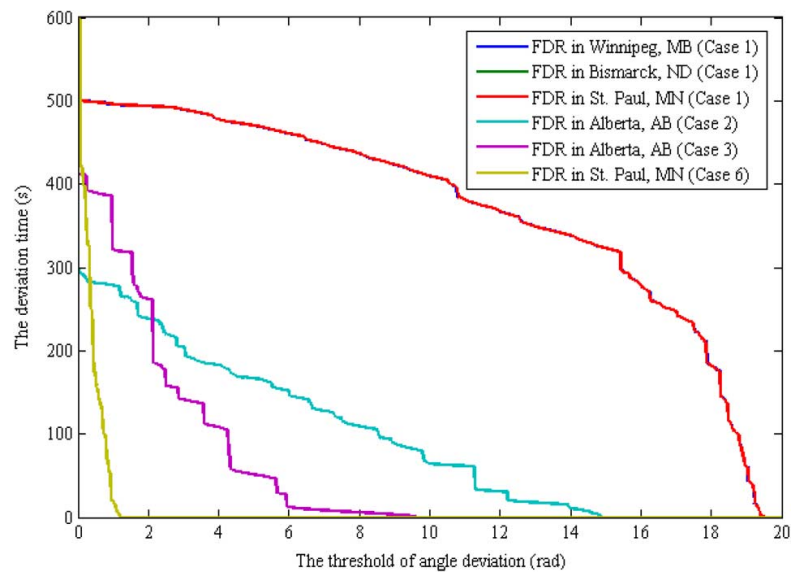

Fig. 26. Sensitivity analysis on the upper threshold of the angle deviation.

viation for cases 1-3 and 6, respectively, which can be employed to determine the upper thresholds of the frequency deviation and angle deviation. It can be seen that the deviation time recorded by the FDR in St. Paul, MN of case 6 is sharply increased if the thresholds of the frequency and angle deviations decline to zero, and the deviation time recorded by the FDRs in Winnipeg, MB, Bismarck, ND and St. Paul, MN of case 1 is more insensitive to the thresholds of the frequency and angle deviations when compared with that of case 6 . Thus, the recorded curve associated with the FDR in St. Paul, MN of case 6 should be used to determine the sensitivity of the thresholds and timer. Figs. 27 and 28 illustrate the thresholds of the frequency deviation and angle deviation against the time of the deviation for cases 4 , 5 and 7-9, respectively, which can be employed to determine the lower thresholds of the frequency deviation and angle deviation. It can be seen that the deviation time recorded by the FDRs in Boston, MA and Bangor, ME of case 7 is more sensitive to the thresholds of the frequency and angle deviations when compared with that of cases 4, 5, 8 and 9 if the threshold of the timer is above one second. Thus, the recorded curve associated with the FDRs in Boston, MA and Bangor, ME of case 7 should be used to determine the sensitivity of the thresholds and timer.

The results of the sensitivity analysis on the thresholds and timers for nine real cases are summarized in Table II, in 


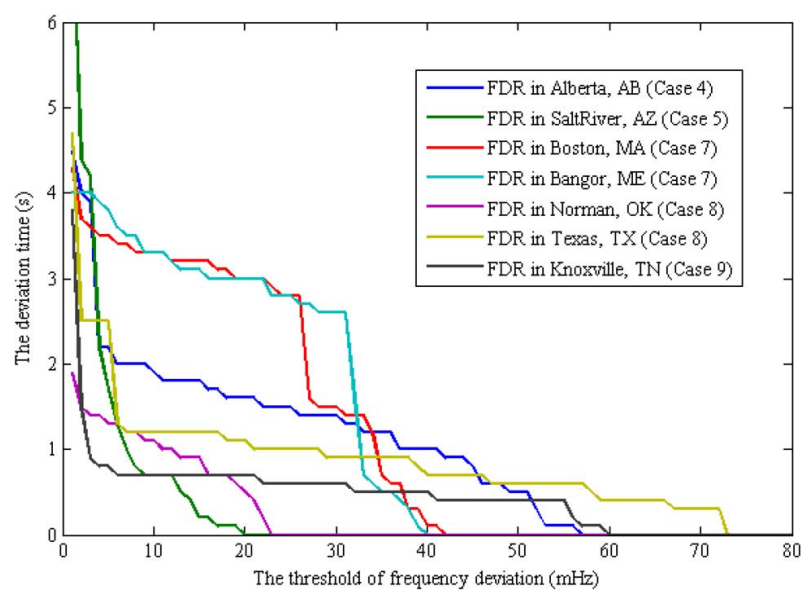

Fig. 27. Sensitivity analysis on the lower threshold of the frequency deviation.

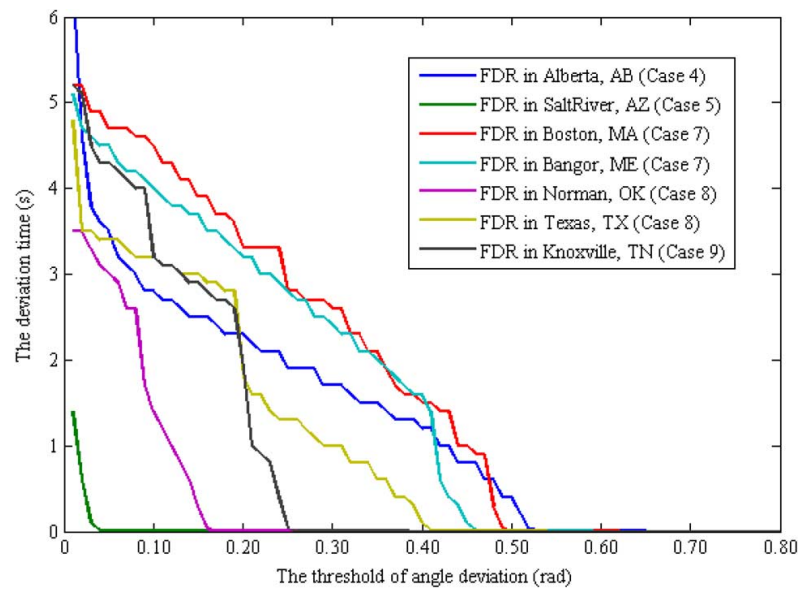

Fig. 28. Sensitivity analysis on the lower threshold of the angle deviation.

TABLE II

RESUlts of SENSITIVITY ANALYSIS ON THE THRESHOLD VALUES

\begin{tabular}{|c|c|c|c|c|}
\hline$T_{\text {th }}(\mathrm{s})$ & $\begin{array}{c}\Delta f_{\text {th, min }} \\
(\mathrm{mHz})\end{array}$ & $\begin{array}{c}\Delta f_{\text {th, max }} \\
(\mathrm{mHz})\end{array}$ & $\begin{array}{c}\theta_{\text {th,min }} \\
(\mathrm{rad})\end{array}$ & $\begin{array}{c}\theta_{\text {th,max }} \\
(\mathrm{rad})\end{array}$ \\
\hline 0.5 & 58 & 87 & 0.48 & 1.19 \\
\hline 1 & 41 & 61 & 0.45 & 1.18 \\
\hline 2 & 31 & 59 & 0.35 & 1.17 \\
\hline 3 & 20 & 56 & 0.24 & 1.16 \\
\hline 4 & 3 & 56 & 0.14 & 1.14 \\
\hline 5 & 2 & 56 & 0.03 & 1.13 \\
\hline 10 & 1 & 53 & 0.01 & 1.06 \\
\hline 50 & 1 & 42 & 0.01 & 0.91 \\
\hline 100 & 1 & 34 & 0.01 & 0.73 \\
\hline
\end{tabular}

which $\Delta f_{\mathrm{th}, \text { min }}, \Delta f_{\mathrm{th}, \text { max }}, \theta_{\mathrm{th}, \text { min }}, \theta_{\mathrm{th}, \text { max }}$ are the minimum and maximum frequency deviation, and the minimum and maximum angle deviation respectively for correct islanding detection under the given time threshold $T_{\mathrm{th}}$. It can be seen that the upper thresholds of the frequency and angle deviations in real cases are insensitive to the threshold of the timer when the threshold of the timer is between 3 and 5 seconds. As a result, it can be concluded that the insensitivity intervals for the thresholds of the timer, frequency deviation and angle deviation are $[3 \mathrm{~s}, 5 \mathrm{~s}],[20 \mathrm{mHz}, 56 \mathrm{mHz}]$ and $[0.24 \mathrm{rad}, 1.13 \mathrm{rad}]$, respectively. So, the thresholds in the real cases can be selected as any values within this insensitivity interval.

It should be pointed out that the angle values recorded may be affected by the transformer connections and phase selection since the measurements are taken from low-voltage singlephase devices. The tap change in the transformers or the change of the phase may lead to the change of the angle measured, which may falsely trigger the islanding detection program when only the change of the angle difference method is used. It may not falsely trigger the islanding detection program by using the frequency difference method. In the FNET, two islanding detection methods, i.e., the frequency difference method and the change of the angle difference method, are jointly used to detect the islanding events in the islanding detection program. As a result, both methods ensure that the transformer connections and phase selection not falsely trigger the islanding detection program. It also should be noted that the proposed islanding detection methods based on wide area measurement system also have a non-detected zone as that in DG systems. When less power flow is transferred through the interconnection transmission lines between islands, the islanding will not lead to large frequency variation and change of angle difference. So the proposed methods cannot be triggered in this circumstance. It is believed that as more and more FDRs of FNET are deployed in North American power grid, the islanding zone of power grid can be detected more precisely, and it can present to all dispatchers a clearer wide-area view of the power grid.

\section{CONCLUSION}

Two islanding detection methods in FNET are proposed, the frequency difference method and the change of angle difference method. The FNET islanding detection program has successfully detected the islanding events since it was installed in the FNET server. The offline simulations based on the measurement data show that FNET islanding detection program does not falsely trigger for generation trip events, load shedding events, or system oscillation events. The insensitivity intervals for the thresholds of the timer, frequency deviation and angle deviation are obtained by sensitivity analysis based on the real recorded cases. The authors envision a suite of intelligent event detection functions that can be enabled by FNET and PMU measurements.

\section{ACKNOWLEDGMENT}

The authors would like to thank Yong Liu, Yong Jia, Penn Markham, Yuming Liu, and Lin Fu who contributed to the success of this effort at the University of Tennessee. The authors also would like to thank their FDR hosts at universities, high schools, companies, and individual residences.

\section{REFERENCES}

[1] Y. C. Zhang, P. Markham, T. Xia, L. Chen, Y. Z. Ye, Z. Y. Wu, Z. Y. Yuan, L. Wang, J. Bank, J. Burgett, R. W. Conners, and Y. Liu, "Widearea frequency monitoring network (FNET) architecture and applications," IEEE Trans. Smart Grid, vol. 1, no. 2, pp. 159-167, Sep. 2010.

[2] Z. A. Zhong, C. C. Xu, B. J. Billian, L. Zhang, S. J. S. Tsai, R. W. Conners, V. A. Centeno, A. G. Phadke, and Y. Liu, "Power system frequency monitoring network (FNET) implementation," IEEE Trans. Power Syst., vol. 20, no. 4, pp. 1914-1921, Nov. 2005.

[3] J. Y. Dong, J. Zuo, L. Wang, K. S. Kook, I. Y. Chung, Y. Liu, S. Affare, B. Rogers, and M. Ingram, "Analysis of power system disturbances based on wide-area frequency measurements," in Proc. 2007 IEEE Power Engineering Society General Meeting, Tampa, FL, USA, Jun. 24-28, 2007, pp. 1-8. 
[4] J. Y. Dong, T. Xia, Y. C. Zhang, T. Weekes, J. S. Thorp, and Y. Liu, "Monitoring power system disturbances at the distribution level," in Proc. 2008 IEEE Power and Energy Society General Meeting, Pittsburgh, PA, USA, Jul. 20-24, 2008, pp. 1-8.

[5] A. Timbus, A. Oudalov, and C. N. M. Ho, "Islanding detection in smart grids," in Proc. 2010 IEEE Energy Conversion Congr. Expo. (ECCE), Atlanta, GA, USA, Sep. 12-16, 2010, pp. 3631-3637.

[6] L. A. C. Lopes and H. L. Sun, "Performance assessment of active frequency drifting islanding detection methods," IEEE Trans. Energy Convers., vol. 21, no. 1, pp. 171-180, Mar. 2006.

[7] L. A. C. Lopes and Y. Z. Zhang, "Islanding detection assessment of multi-inverter systems with active frequency drifting methods," IEEE Trans. Power Del., vol. 23, no. 1, pp. 480-486, Jan. 2008.

[8] G. K. Hung, C. C. Chang, and C. L. Chen, "Automatic phase-shift method for islanding detection of grid-connected photovoltaic inverters," IEEE Trans. Energy Convers., vol. 18, no. 1, pp. 169-173, Mar. 2003.

[9] W. C. Wang, J. Kliber, and W. Xu, "A scalable power-line-signalingbased scheme for islanding detection of distributed generators," IEEE Trans. Power Del., vol. 24, no. 2, pp. 903-909, Apr. 2009.

[10] X. Y. Wang, W. Freitas, and W. Xu, "Dynamic non-detection zones of positive feedback anti-islanding methods for inverter-based distributed generators," IEEE Trans. Power Del., vol. 26, no. 2, pp. 1145-1155, Apr. 2011.

[11] G. Y. Xu, V. Vittal, A. Meklin, and J. E. Thalman, "Controlled islanding demonstrations on the WECC system," IEEE Trans. Power Syst., vol. 26, no. 1, pp. 334-343, Feb. 2011.

[12] G. Y. Xu and V. Vittal, "Slow coherency based cut set determination algorithm for large power systems," IEEE Trans. Power Syst., vol. 25, no. 2, pp. 877-884, May 2010.

[13] N. Senroy, G. T. Heydt, and V. Vittal, "Decision tree assisted controlled islanding," IEEE Trans. Power Syst., vol. 21, no. 4, pp. 1790-1797, Nov. 2006.

[14] B. Yang, V. Vittal, and G. T. Heydt, "Slow-coherency-based controlled islanding - A demonstration of the approach on the August 14, 2003 blackout scenario," IEEE Trans. Power Syst., vol. 21, no. 4, pp. $1840-1847$, Nov. 2006.

[15] H. You, V. Vittal, and X. M. Wang, "Slow coherency-based islanding," IEEE Trans. Power Syst., vol. 19, no. 1, pp. 483-491, Feb. 2004.

[16] H. You, V. Vittal, and Z. Yang, "Self-healing in power systems: An approach using islanding and rate of frequency decline based load shedding," IEEE Trans. Power Syst., vol. 18, no. 1, pp. 174-181, Feb. 2003.

[17] K. Sun, D. Z. Zheng, and Q. Lu, "Searching for feasible splitting strategies of controlled system islanding," Proc. Inst. Elect. Eng., Gen., Transm., Distrib., vol. 153, no. 1, pp. 89-98, Jan. 2006.

[18] K. Sun, D. Z. Zheng, and Q. Lu, "A simulation study of OBDD-based proper splitting strategies for power systems under consideration of transient stability," IEEE Trans. Power Syst., vol. 20, no. 1, pp. 389-399, Feb. 2005.

[19] Q. C. Zhao, K. Sun, D. Z. Zheng, J. Ma, and Q. Lu, "A study of system splitting strategies for island operation of power system: A two-phase method based on OBDDs," IEEE Trans. Power Syst., vol. 18, no. 4, pp. 1556-1565, Nov. 2003.

[20] K. Sun, D. Z. Zheng, and Q. Lu, "Splitting strategies for islanding operation of large-scale power systems using OBDD-based methods," IEEE Trans. Power Syst., vol. 18, no. 2, pp. 912-923, May 2003.

[21] W. X. Liu, L. Liu, and D. A. Cartes, "Slow coherency and angle modulated particle swarm optimization based islanding of large scale power systems," in Proc. 2007 Int. Joint Conf. Neural Networks (IJCNN 2007), Orlando, FL, USA, Aug. 12-17, 2007, pp. 2087-2092.

Zhenzhi Lin received the B.E. degree from Hefei University of Technology, China, in 2002, and the Ph.D. degree from South China University of Technology, China, in 2008, both in electrical engineering.

He was a postdoctoral researcher in the Min Kao Department of Electrical Engineering and Computer Science at the University of Tennessee, Knoxville, TN, USA, from 2010 to 2011 . He is currently a faculty in the School of Electrical Engineering, Zhejiang University, China. His research interests include power system wide area monitoring and control, and power system restoration.

Tao Xia received the B.S. degree from Xi' an University of Technology, China, in 2000, the M.S. degree from Huazhong University of Science and Technology, China, in 2003, and the Ph.D. degree from Virginia Polytechnic Institute and State University, Blacksburg, VA, USA, in 2009, respectively.

His interests involve wide-area power system monitoring and control, and power system dynamic analysis.
Yanzhu Ye received the B.S. and M.S. degrees from Tsinghua University, China, in 2005 and 2007, respectively, and the Ph.D. degree from the University of Tennessee, Knoxville, TN, USA.

Her interests include wide-area power system monitoring, and power system dynamic analysis.

Ye Zhang received the B.S. degrees in software engineering from Shanghai Jiaotong University, China, in 2006, and the M.S. degree in computer engineering from Polytechnic University of Milan, Italy, in 2008.

She is currently pursuing the Ph.D. degree in the Min H. Kao Department of Electrical Engineering and Computer Science, University of Tennessee, Knoxville, TN, USA. Her research interest lies in the visualization study of power system dynamics.

Lang Chen received the B.S. and M.S. degrees from North China Electric Power University in 2005 and 2008, respectively, and the Ph.D. degree from the University of Tennessee, Knoxville, TN, USA.

Her interests involve wide-area power system monitoring and power system dynamic analysis.

Yilu Liu (S'88-M'89-SM'99-F'04) is currently the Governor's Chair at the University of Tennessee, Knoxville, TN, USA, and Oak Ridge National Laboratory. Prior to joining UTK/ORNL, she was a Professor at Virginia Tech. She led the effort to create the North America power grid monitoring network (FNET) at Virginia Tech which is now operated at UTK and ORNL as GridEye. Her current research interests include power system wide-area monitoring and control, large interconnection level dynamic simulations, and power transformer modeling and diagnosis.

Kevin Tomsovic (S'81-M'88-SM'00-F'07) received the B.S. degree from Michigan Technical University, Houghton, MI, USA, in 1982, and the M.S. and Ph.D. degrees from University of Washington, Seattle, WA, USA, in 1984 and 1987, respectively, all in electrical engineering.

He is currently CTI Professor and Head of the Department of Electrical Engineering and Computer Science at the University of Tennessee, Knoxville, TN, USA.

Terry Bilke received the M.B.A. degree from the University of Wisconsin, Whitewater, WI, USA, in 1990 and the M.S. degree in industrial engineering from Colorado State University, Fort Collins, CO, USA, in 1998 and the Ph.D. degree in management of technology from Indiana State University, Terre Haute, IN, USA, in 2006.

$\mathrm{He}$ is Director of NERC Standards and Compliance at the Midwest ISO, Carmel, IN, USA. Prior to the Midwest ISO, he was a power system operator at Wisconsin Electric Power Company. He is chairman of the NERC Resources subcommittee and Operations Implementation co-chair for NASPI.

Fushuan Wen received the B.E. and M.E. degrees in electrical engineering from Tianjin University, Tianjin, China, in 1985 and 1988, respectively, and the Ph.D. degree from Zhejiang University, Zhejiang, China, in 1991.

He joined the faculty of Zhejiang University in 1991, and has been a full professor and the director of the Institute of Power Economics and Information since 1997, and the director of Zhejiang University-Insigma Joint Research Center for Smart Grids since 2010. He had been a university distinguished professor, the deputy dean of the School of Electrical Engineering and the director of the Institute of Power Economics and Electricity Markets in South China University of Technology (SCUT), China, from 2005 to 2009. His current research interests lie in power industry restructuring, power system alarm processing, fault diagnosis and restoration strategies, as well as smart grids. 